\title{
Antibacterial and antifungal activities of new acylated derivatives of epigallocatechin gallate
}

\author{
Yoshimi Matsumoto ${ }^{*}$, Kunihiro Kaihatsu ${ }^{2 *}$, Kunihiko Nishino $^{3}$, Miho Ogawa $^{4}$, Nobuo Kato ${ }^{2}$ and \\ Akihito Yamaguchi ${ }^{1,5}$
}

${ }^{1}$ Department of Cell Membrane Biology, Institute of Scientific and Industrial Research, Osaka University, Ibaraki, Osaka, Japan

${ }^{2}$ Department of Organic Fine Chemicals, Institute of Scientific and Industrial Research, Osaka University, Ibaraki, Osaka, Japan

${ }^{3}$ Laboratory of Microbiology and Infectious Diseases, Institute of Scientific and Industrial Research, Osaka University, Ibaraki, Osaka, Japan

${ }^{4}$ Department of Bacteriology, Bio Medical Laboratories, Inc., Kawagoe, Saitama, Japan

${ }^{5}$ Graduate School of Pharmaceutical Sciences, Osaka University, Suita, Osaka, Japan

\section{Edited by:}

Jose L. Martinez, Centro Nacional de Biotecnología, Spain

\section{Reviewed by:}

Balazs Libisch, Research Institute for Soil Science and Agricultural

Chemistry of Hungarian Academy of

Sciences, Hungary

Lilia Macovei, Harvard Medical

School, USA

\section{*Correspondence:}

Yoshimi Matsumoto, Department of Cell Membrane Biology, Institute of Scientific and Industrial Research, Osaka University, 8-1 Mihogaoka, Ibaraki, Osaka 567-0047, Japan. e-mail:yoshimi@sanken.osaka-u.ac.jp; Kunihiro Kaihatsu, Department of Organic Fine Chemicals, Institute of Scientific and Industrial Research, Osaka University, 8-1 Mihogaoka, Ibaraki, Osaka 567-0047, Japan. e-mail:kunihiro@sanken.osaka-u.ac.jp
(-)-Epigallocatechin-3-O-gallate (EGCG) has useful antiviral, antimicrobial, antitoxin, and antitumor properties. Previously, Mori et al. (2008) found that addition of long acyl chains (C16-18) to EGCG enhanced its anti-influenza virus activity up to 44-fold. The chemical stability of EGCG against oxidative degradation was also enhanced by acylation. We further evaluated the in vitro activity spectrum of the EGCG derivatives against a wide range of bacteria and fungi. A series of EGCG $O$-acyl derivatives were synthesized by lipase-catalyzed transesterification. These derivatives exhibited several-fold higher activities than EGCG, particularly against Gram-positive organisms. Antifungal MICs of the derivatives were also two to fourfold lower than those of EGCG. The activities of the EGCG derivatives against Gram-negative bacteria were not distinguishable from those of EGCG. Among the derivatives evaluated, MICs of dioctanoate and palmitate (C16) for 17 Staphylococcus aureus strains were $4-32 \mu \mathrm{g} / \mathrm{ml}$, although MIC of EGCG for these 17 strains was $\geq 128 \mu \mathrm{g} / \mathrm{ml}$. C16 demonstrated rapid bactericidal activity against methicillin-resistant $S$. aureus (MRSA) ATCC43300 at $\geq 16 \mu \mathrm{g} / \mathrm{ml}$. The enhanced activity of C16 against $S$. aureus was supported by its increased membrane-permeabilizing activity determined by increased SYTOX Green uptake. The EGCG derivatives were exported in Escherichia coli using the efflux pump AcrAB-TolC. The tolC deletion mutant exhibited higher sensitivity to EGCG and the derivatives than wild-type. Addition of long alkyl chains to EGCG significantly enhanced its activities against several bacteria and fungi, particularly against $S$. aureus including MRSA. C16 might potentially become under specified circumstances an alternative or supplement to antibiotics and disinfectants in the future.

Keywords: epigallocatechin gallate, fatty acid esters, bactericidal activity Staphylococcus aureus

\section{INTRODUCTION}

Green tea has long been a daily food in Japan with beneficial health effects. Antibacterial and antitoxin effects of tea (Camellia sinensis) leaves are already proved in Japan in 1930s (Shimamura, 2009). After World War II, anti-influenza virus activity of tea extracts was published in 1949 (Green, 1949), and antibacterial activity of tea was published in India (Das, 1962). More than a quarter of a century later, antibacterial activity of tea was eventually rediscovered in 1989 (Toda et al., 1989). Since then, recent studies proved that polyphenol components of tea leaves have antitoxin (Hara et al., 1990; Toda et al., 1990; Hisano et al., 2003), antiviral (Mukoyama et al., 1991; Nakayama et al., 1993; Yamaguchi et al., 2002), and antitumor properties (Mukhtar et al., 1992; Shimamura and Hara, 1997) other than antimicrobial activity (Toda et al., 1989; Yam et al., 1997). Tea polyphenols have broad antibacterial spectrum, and inhibit various kinds of pathogenic bacteria such as Bacillus cereus, Campylobacter spp., Clostridium perfringens, Escherichia coli O157, Helicobacter pylori, Legionella pneumophila, Mycobacterium tuberculosis, Mycoplasma pneumonia, Proteus vulgaris, Pseudomonas aeruginosa, Salmonella spp., Serratia marcescens, Staphylococcus spp., oral pathogenic Streptococci (Friedman, 2007). In polyphenols, catechins belong to the flavan-3-ol class of flavonoids. Catechins are thermo stable and stable in acidic condition although those are unstable in neutral and alkali condition. (-)-Epigallocatechin-3-O-gallate (EGCG) is the major polyphenolic compound found in green tea and it has the strongest activity in various useful properties. However, although antiviral properties of tea polyphenols have been demonstrated also in vivo (Xiao et al., 2008), EGCG is not so useful for clinical use because of its poor membrane permeability, low chemical stability, and rapid metabolism. Previously, we synthesized a series of EGCG fatty acid monoester derivatives by lipase-catalyzed transesterification and found that addition of long acyl chains (C16-18) enhanced the anti-influenza virus activity of EGCG up to 44-fold (Mori et al., 2008). Acylation also enhanced the in vitro antitumor activities of EGCG in an alkyl chain lengthdependent manner (Matsumura et al., 2008). Furthermore, the chemical stability of EGCG in tissue culture medium was enhanced 
10-fold by acylation (Data not shown). But effects of acylation on antimicrobial and antitoxin activities of EGCG have not yet been evaluated until now. In this study, we additionally synthesized unsaturated fatty acid esters and dioctanoyl esters, and evaluated the in vitro activities of the acylated EGCG derivatives against a wide range of bacteria and fungi. These derivatives exhibited increased antimicrobial activities, particularly against Staphylococcus aureus and some fungal species. The antibacterial activity of palmitate was evaluated in detail. Taken together, these data show a potential use of EGCG $O$-acyl derivatives as novel antimicrobial agents.

\section{MATERIALS AND METHODS}

\section{SYNTHESIS OF EGCG $\boldsymbol{O}$-ACYL DERIVATIVES}

A series of EGCG $O$-acyl derivatives (Figure 1; Table 1) were synthesized by lipase-catalyzed transesterification, according to

$$
\text { (c) }
$$

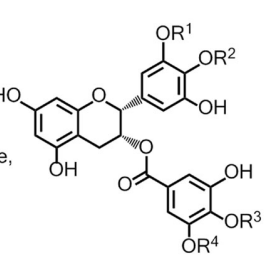

EGCG-fatty acid esters
FIGURE 1 | Lipase-catalyzed synthesis of EGCG fatty acid esters. One or two of $\mathrm{R}^{1-4}$ are substituted with fatty acids. a method reported previously (Mori et al., 2008). This method yielded B-ring-modified esters as the major products except linolenate ester (C18TE). In case of synthesis of saturated fatty acid monoesters, the ratio of regioisomers was consistent regardless of the alkyl chain length. Unsaturated fatty acid esters and dioctanoyl esters $(\mathrm{C} 8 \times 2)$ were also synthesized, and the ratio of their regioisomers is summarized in Table 1. Mixtures of these isomers were used without isolation because they have similar chemical properties and the antiviral activities of these mixtures did not vary with their composition ratio (Mori et al., 2008). Log $P$ values were calculated by a software; ChemBioDraw Ultra ver. 11 and used as lipophilicity index (Table 1). Higher $\log P$ means higher lipophilicity. The derivatives were dissolved in DMSO and diluted with distilled water.

\section{BACTERIAL STRAINS}

In total, $17 S$. aureus strains [9 methicillin-sensitive $S$. aureus (MSSA) and 8 methicillin-resistant $S$. aureus (MRSA) strains]. Five MSSA and seven MRSA strains were clinically isolated from Japan (Table 2). E. coli MG1655 (wild-type) and its efflux pump gene deletion mutants, $\triangle a c r B, \Delta a c r D$, $\triangle a c r E F$, and $\triangle$ tolC constructed by phage P1-mediated transduction (Davis et al., 1980; Nishino et al., 2008) were used to evaluate the effects of efflux pumps on the susceptibility of E. coli to EGCG. Moreover, standard strains of various bacterial and fungal species (Tables 3-5) were also analyzed.

\begin{tabular}{|c|c|c|c|}
\hline Abbreviation & Acyl group & $\begin{array}{l}\text { Position of acyl group on EGCG } \\
\text { fatty acid esters }(\%)^{a} ; R^{1}: R^{2}: R^{3}: R^{4}\end{array}$ & $\log P^{c}$ \\
\hline EGCG & $\begin{array}{c}\text { None } \\
\mathrm{O}\end{array}$ & & 2.07 \\
\hline C8 (octanoate) & & $35: 39: 6: 20$ & 4.79 \\
\hline C12 (laurate) & & $30: 39: 9: 22$ & 6.46 \\
\hline C16 (palmitate) & & $38: 35: 7: 20$ & 8.13 \\
\hline C16E (palmitoleate) & & $28: 37: 9: 26$ & 7.81 \\
\hline C18 (stearate) & & $38: 35: 7: 20$ & $8.97^{*}$ \\
\hline C18TE (linolenate) & & $15: 19: 4: 62$ & 8.01 \\
\hline C20 (eicosanoate) & & $28: 37: 9: 26$ & $9.80 *$ \\
\hline
\end{tabular}

Table 1 | EGCG fatty acid esters and the ratio of their regioisomers. 
Table 2 | MICs of EGCG derivatives for S. aureus.

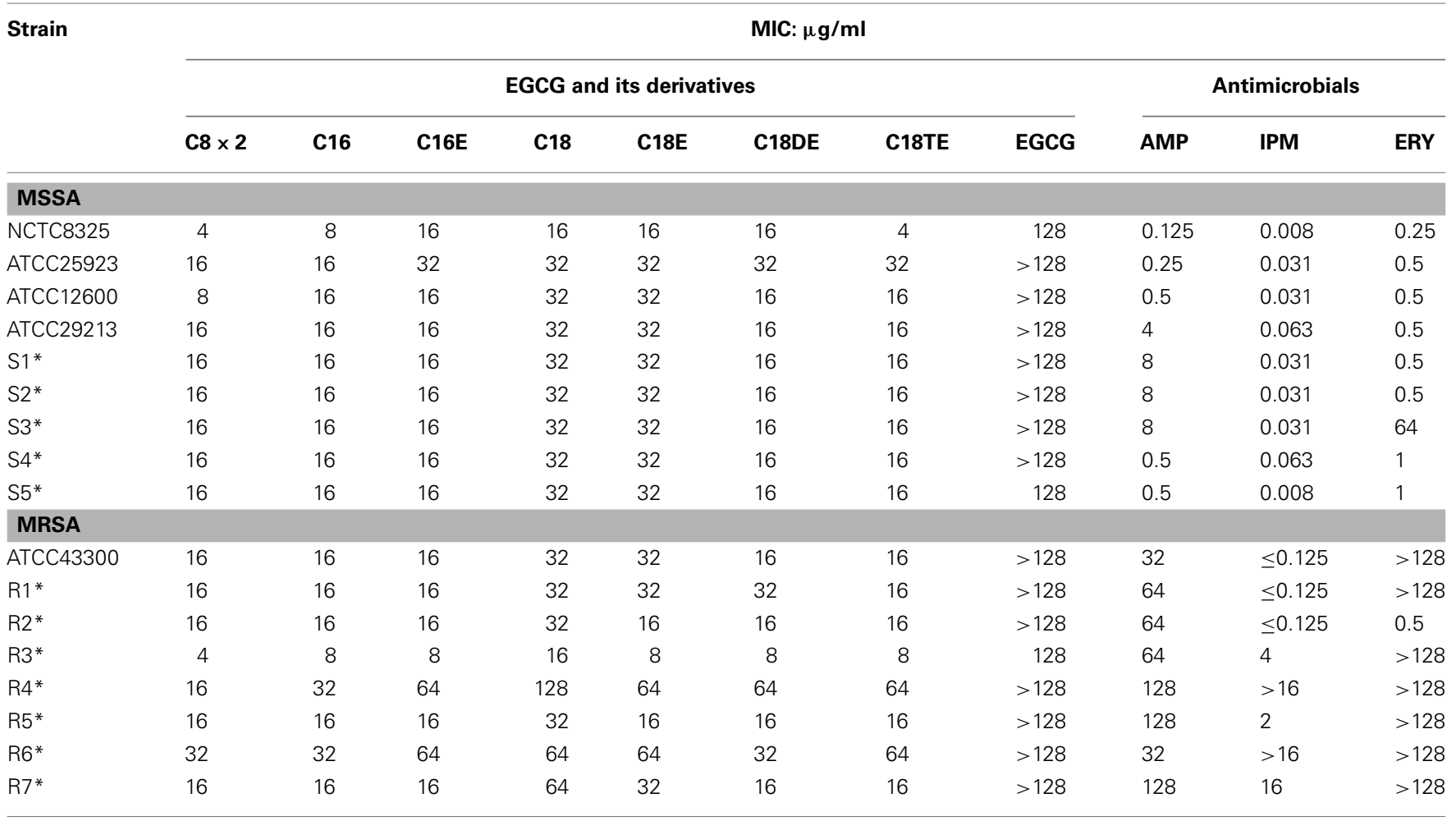

${ }^{*}$ Clinical isolates from Japan.

Table 3 | Antibacterial activities of C16 against Gram-positive bacteria.

Organism

(MIC: $\mu \mathrm{g} / \mathrm{ml}$ )

\begin{tabular}{lccccc} 
& C16 & EGCG & AMX & CFZ & ERY \\
\hline Bacillus subtilis ATCC6051 & 16 & 64 & 0.06 & 0.25 & $\leq 0.03$ \\
Bacillus cereus ATCC14579 & 16 & 64 & 2 & 64 & 0.06 \\
Staphylococcus epidermidis ATCC14990 & 8 & 128 & 0.125 & 0.5 & 0.125 \\
Streptococcus pneumoniae ATCC49619 & 16 & 32 & $\leq 0.03$ & 1 & 0.5 \\
Streptococcus pyogenes ATCC19615 & 64 & 128 & $\leq 0.03$ & 0.25 & $\leq 0.03$ \\
Streptococcus agalactiae ATCC13813 & $>64$ & $>128$ & $\leq 0.03$ & 0.125 & $\leq 0.03$ \\
Enterococcus faecalis ATCC29212 & 16 & 256 & 0.25 & 32 & 0.125 \\
Micrococcus luteus ATCC9341 & 32 & 128 & 0.06 & 2 & 0.25 \\
\hline
\end{tabular}

\section{ANTIBACTERIAL AGENTS AND CHEMICALS}

Ampicillin (AMP), amoxicillin (AMX), cefazolin (CFZ), and vancomycin (VAN) were purchased from Sigma-Aldrich Co. (St Louis, USA). Imipenem (IPM, Banyu Pharmaceutical Co., Tokyo, Japan), erythromycin (ERY, Nacalai Tesque Inc., Kyoto, Japan), teicoplanin (TEC, Astellas Pharma Inc., Tokyo, Japan), and nisin (MP Biomedicals, LLC, Illkirch, France) were also used. Fluconazole (FLC, Sigma-Aldrich Co.) was used for experiments with fungi.

\section{DETERMINATION OF MICs}

MICs were determined by the micro broth dilution method using Mueller-Hinton broth (Difco, Becton, Dickinson, and Company
Japan, Tokyo, Japan) according to guidelines M07-A8 of the Clinical and Laboratory Standards Institute (2009; CLSI, Wayne, PA, USA). $\mathrm{OD}_{600}$ was determined using a microplate reader after overnight incubation. For Figure 2, average and SD of $\mathrm{OD}_{600}$ of four wells for each concentration were calculated. RPMI 1640 medium (Sigma-Aldrich Co.) buffered with 0.165 M 3-(Nmorpholino) propane sulfonic acid at $\mathrm{pH} 7.0$ was used for fungi.

Effects of peptidoglycan from S. aureus, D-Ala-D-Ala, and lipopolysaccharide (LPS; all purchased from Sigma-Aldrich Co.) on the antistaphylococcal activities of $\mathrm{C} 16$ were evaluated by comparison of MIC with or without $30 \mu \mathrm{g} / \mathrm{ml}$ of each bacterial cell component according to the literature (Zhao et al., 2001). 
Table 4 | Antibacterial activities of C16 against Gram-negative bacteria.

Organism

\begin{tabular}{|c|c|c|c|c|c|c|}
\hline & & & & & & \\
\hline & C16 & EGCG & AMX & CFZ & ERY & VAN \\
\hline Neisseria meningitidis* & 16 & 32 & 0.25 & 0.5 & 0.25 & 2 \\
\hline Neisseria gonorrhoeae ATCC49226 & 16 & 32 & 0.5 & 4 & 4 & 32 \\
\hline Haemophilus influenzae ATCC49766 & $>64$ & $>256$ & 0.5 & 2 & 8 & $>64$ \\
\hline Moraxella catarrhalis* & 64 & 128 & 0.25 & 0.5 & 0.125 & 64 \\
\hline Escherichia coli ATCC25922 & $>64$ & $>256$ & 8 & 2 & 64 & $>64$ \\
\hline Klebsiella pneumoniae ATCC13883 & $>64$ & $>256$ & $>64$ & 2 & 64 & $>64$ \\
\hline Klebsiella oxytoca ATCC8724 & $>64$ & $>256$ & 64 & 2 & 128 & $>64$ \\
\hline Citrobacter freundii ATCC8090 & $>64$ & $>256$ & 64 & 32 & 128 & $>64$ \\
\hline Enterobacter cloacae ATCC13047 & $>64$ & $>256$ & $>64$ & $>128$ & $>128$ & $>64$ \\
\hline Enterobacter aerogenes ATCC13048 & $>64$ & $>256$ & $>64$ & 128 & 128 & $>64$ \\
\hline Proteus mirabilis ATCC29906 & $>64$ & $>256$ & 0.5 & 4 & $>128$ & $>64$ \\
\hline Proteus vulgaris ATCC13315 & $>64$ & $>256$ & 16 & 32 & $>64$ & $>64$ \\
\hline Serratia marcescens ATCC13880 & $>64$ & $>256$ & 64 & $>64$ & 64 & $>64$ \\
\hline Salmonella typhimurium IID1000 & $>64$ & $>256$ & 0.5 & 1 & 64 & $>64$ \\
\hline Yersinia enterocolitica ATCC9610 & $>64$ & $>256$ & 4 & 1 & 32 & $>64$ \\
\hline Aeromonas hydrophila ATCC7966 & $>64$ & $>256$ & $>64$ & 32 & 16 & $>64$ \\
\hline Pseudomonas aeruginosa ATCC7700 & $>64$ & $>256$ & $>64$ & $>128$ & 128 & $>64$ \\
\hline Pseudomonas fluorescence ATCC8750 & $>64$ & $>256$ & 8 & 16 & 32 & $>64$ \\
\hline Stenotrophomonas maltophilia ATCC13637 & $>64$ & $>256$ & $>64$ & 128 & 16 & $>64$ \\
\hline Campylobacter jejuni ATCC33291 & 8 & 8 & 4 & $>64$ & 2 & $>64$ \\
\hline Helicobacter pylori ATCC43504 & 16 & 16 & $\leq 0.03$ & 0.25 & 0.125 & $>64$ \\
\hline
\end{tabular}

${ }^{*}$ A clinical isolates from Japan.

(MIC: $\mu \mathrm{g} / \mathrm{ml}$ )

Table 5 | Antifungal activities of EGCG derivatives.

\begin{tabular}{|c|c|c|c|c|c|c|c|c|c|}
\hline \multirow[t]{2}{*}{ Organism } & \multicolumn{9}{|c|}{ EGCG and its derivatives (MIC: $\mu \mathrm{g} / \mathrm{mI}$ ) } \\
\hline & $\mathrm{C8} \times 2$ & C16 & C16E & C18 & C18E & C18DE & C18TE & EGCG & Fluconazole \\
\hline Candida albicans ATCC24433 & 32 & 32 & 16 & 16 & 16 & 16 & 16 & 64 & 0.25 \\
\hline Candida parapsilosis ATCC22019 & 16 & 16 & 16 & 16 & 16 & 16 & 16 & 64 & 2 \\
\hline Candida tropicalis ATCC750 & 32 & 32 & 16 & 32 & 32 & 32 & 16 & 64 & 2 \\
\hline Candida krusei ATCC6258 & 16 & 16 & 16 & 16 & 16 & 16 & 16 & 64 & 32 \\
\hline Candida glabrata ATCC90030 & 16 & 16 & 16 & 16 & 16 & 32 & 32 & 32 & 16 \\
\hline Cryptococcus neoformans ATCC90112 & 16 & 8 & 8 & 16 & 16 & 8 & 8 & 64 & 1 \\
\hline Aspergillus fumigatus* & 32 & 32 & 16 & 32 & 32 & 32 & 16 & 64 & $>64$ \\
\hline
\end{tabular}

${ }^{*}$ A clinical isolate from Japan.

The combinatorial effects of C16 or EGCG with IPM or AMP on MRSA were determined by the checkerboard method (Martinez-Irujo et al., 1996; Hu et al., 2001). The fractional inhibitory concentration (FIC) indices were calculated as follows: $\mathrm{FIC}=\left[\mathrm{MIC}_{\mathrm{A}}\right.$ in combination $/ \mathrm{MIC}_{\mathrm{A} \text { alone }}+\mathrm{MIC}_{\mathrm{B} \text { in combination }} /$ $\mathrm{MIC}_{\mathrm{B} \text { alone }}$ ]. Synergy was defined as an FIC index of less than 0.5 and addition as an FIC index of 0.5-1.0.

\section{DETERMINATION OF KILLING ACTIVITIES}

Killing curves of C16 against MRSA ATCC43300, E. coli MG1655, and $P$. aeruginosa PAO1 were plotted and evaluated. Bacterial suspensions were diluted to approximately $1.0 \times 10^{5} \mathrm{CFUs} / \mathrm{ml}$ in Mueller-Hinton broth. Bacterial counts were determined at selected time intervals of $0,2,4$, and $6 \mathrm{~h}$ by enumerating the colonies formed after plating 10 -fold serially diluted specimens of $100 \mu \mathrm{l}$ aliquots on two Mueller-Hinton agar plates for each dilution; the plates were incubated at $37^{\circ} \mathrm{C}$. The average numbers and SD of two to four plates were calculated.

\section{SYTOX GREEN UPTAKE ASSAY TO DETERMINE MEMBRANE DAMAGE}

Exponentially growing cells of MSSA ATCC25923 and MRSA ATCC43300 harvested in phosphate-buffered saline were used. SYTOX Green (Lonza Walkersville, Inc., USA) is a high-affinity nucleic acid stain that can easily penetrate cells with compromised 
membranes but not those with intact membranes. Membrane integrity was determined after exposure to C16, EGCG or nisin for 15 min by measuring SYTOX Green uptake. Using the plate reader, fluorescence was determined (Ex/Em: 504/523 nm) after 10 min incubation with SYTOX Green in a black 384-well plate (Matsumoto et al., 2011). The means and SD values of triple data were calculated.

\section{RESULTS}

\section{RELATIONSHIP BETWEEN ACYL CHAIN LENGTH AND ACTIVITIES OF EGCG 0-ACYL DERIVATIVES AGAINST S. AUREUS}

Structures of the EGCG $O$-acyl derivatives and the ratio of each acyl component are listed in Figure 1 and Table 1. S. aureus is one of the most sensitive organisms to tea extract (Toda et al., 1989). The effect of acyl chain length on antistaphylococcal activity was evaluated (Figure 2) on the basis of the results of a previous study on antiviral activities of the acylated EGCG derivatives against influenza virus. These antiviral activities were increased in an acyl chain length-dependent manner (Mori et al., 2008). Growth inhibitory activities of EGCG for MSSA ATCC25923 and MRSA ATCC43300 were not increased by C8, but were increased by acyl chains longer than $\mathrm{C} 12$. However, these activities decreased in the derivatives with an acyl chain length longer than C18. C12 and C16 showed the strongest activity (MIC; $8 \mu \mathrm{g} / \mathrm{ml}$ ) against both ATCC25923 and ATCC43300 strains. We then synthesized C8 $\times 2$, C16E, C18E, C18DE, and C18TE and evaluated the effect of diacyl as well as unsaturated acyl chains against 17 S. aureus strains including 8 MRSA strains (Table 2). Although most of these strains had similar sensitivities to the EGCG derivatives, some MRSA strains, particularly IPM-resistant strains, exhibited higher MICs. $\mathrm{C} 8 \times 2$ was similar to $\mathrm{C} 16$ and had the strongest activity among this set of derivatives. In C18 series of derivatives, unsaturation of fatty acid moieties increased the antimicrobial activity, although this effect was not evident in $\mathrm{C} 16$. The $\log P$ values of these derivatives indicated that water solubility improved by the introduction of an unsaturated bond into C18 (Table 1). Among the derivatives evaluated, MICs of $\mathrm{C} 8 \times 2$ and $\mathrm{C} 16$ for all the 17 strains were $\leq 32 \mu \mathrm{g} / \mathrm{ml}$, although MICs of EGCG for these strains were $\geq 128 \mu \mathrm{g} / \mathrm{ml}$.

\section{ANTIMICROBIAL ACTIVITIES OF C16 AGAINST VARIOUS SPECIES}

Antibacterial and antifungal activities of $\mathrm{C} 16$ are presented in Tables 3-5. Table 3 shows MICs for Gram-positive organisms other than S. aureus. C16 showed several-fold higher activities than EGCG against these microbial strains except Streptococci, against which there was less than twofold difference between MICs of both compounds. C16 exhibited 16-fold superior activities against $S$. epidermidis and Enterococcus faecalis than EGCG. Suppression of growth of Gram-negative bacteria was barely detected at the highest concentrations of C16 or EGCG (Table 4), making it difficult to distinguish the differences in their activities based on their MICs. While Gram-negative bacteria tend to be insensitive to EGCG, $C$. jejuni and H. pylori were highly sensitive to EGCG and C16. Neisseria meningitidis and $N$. gonorrhoeae also exhibited sensitivity to $\mathrm{C} 16$.

Antifungal activity of EGCG and its acyl derivatives were evaluated against various species of Candida, Cryptococcus neoformans, and Aspergillus fumigatus (Table 5). MICs of the derivatives for these fungi were two to fourfold superior to those of EGCG. C16E exhibited the highest activities against these fungi. Introduction of unsaturated bonds into the acyl chain tended to increase the antifungal activities of the derivatives.

\section{KILLING ACTIVITIES OF C16}

The rapid bactericidal activity of tea extracts against $S$. aureus are known through research on the antibacterial activities of catechins (Toda et al., 1989). A time-kill study was conducted against MRSA ATCC43300, E. coli MG1655, and P. aeruginosa PAO1 (Figure 3). C16 demonstrated rapid bactericidal activity against MRSA at $\geq 16 \mu \mathrm{g} / \mathrm{ml}$. However, $128 \mu \mathrm{g} / \mathrm{ml}$ of C16 only showed
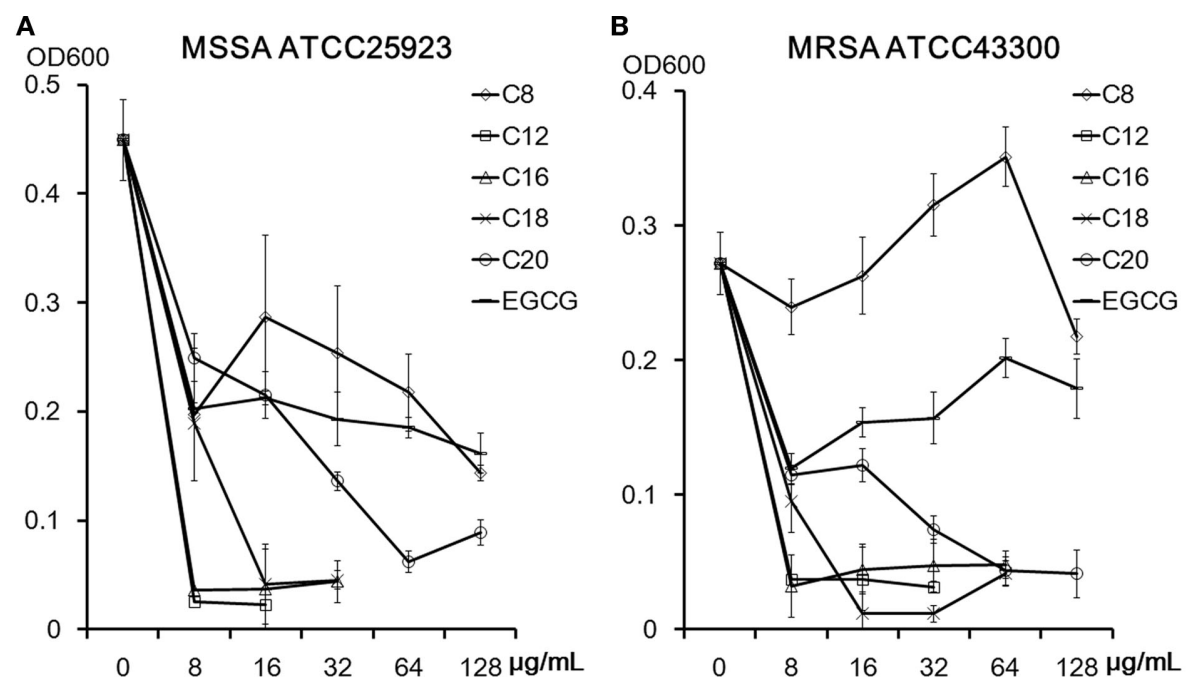

FIGURE 2 | Acyl chain length of EGCG derivatives and their antistaphylococcal activities against MSSA ATCC25923 (A) and MRSA ATCC43300 (B). Mean $\mp$ SD of each four wells optical density $(600 \mathrm{~nm})$ after $18 \mathrm{~h}$ incubation are shown. 

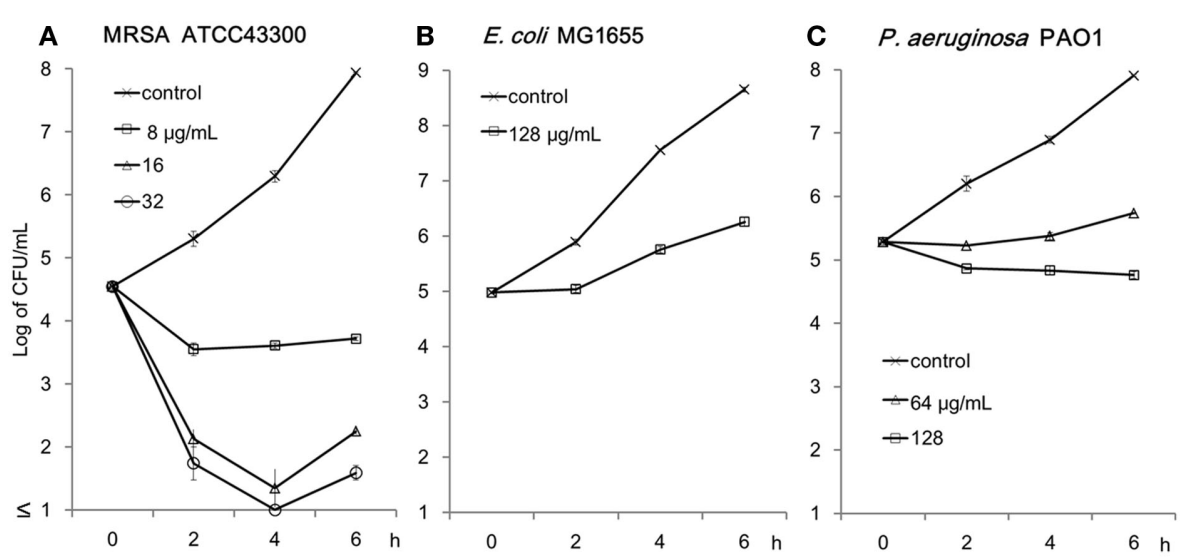

FIGURE 3 | Killing activity of C16 against MRSA ATCC43300 (A), E. coli MG1655 (B), and $P$ aeruginosa PAO1 (C). Cells were incubated with C16 in Muller-Hinton broth at $37^{\circ} \mathrm{C}$ on a shaker. Residual cells after 2, 4, and $6 \mathrm{~h}$ incubation were counted as colonies grown on the Mueller-Hinton agar plates. Mean $\mp$ SD of the residual CFUs calculated from two to four plates are shown. bacteriostatic activity against E. coli MG1655 and P. aeruginosa PAO1.

\section{COMBINATORIAL EFFECTS OF C16 ON ANTIBACTERIAL ACTIVITY OF $\beta$-LACTAMS IN THE CHECKERBOARD METHOD}

The synergistic effects of EGCG with $\beta$-lactams have been well described (Hu et al., 2001, 2002a). C16 and EGCG were evaluated in combination with IPM or AMP against MRSA strains (Figures 4 and 5). We used two MRSA strains, IPM-sensitive ATCC43300 and IPM-resistant clinical isolate R3. C16 enhanced the activities of IPM and AMP against both MRSA strains (Figure 4); the FIC indices were 0.5-1.0. On the other hand, EGCG exhibited negative activity with IPM against IPM-sensitive ATCC43300 (Figure 5A), although synergy between EGCG and IPM (FIC index $<0.07$ ) against IPM-resistant strain R3 was remarkable (Figure 5B). EGCG also showed pronounced synergistic activity with AMP against both MRSA strains (FIC indices $<0.188$; Figures 4C,D). In other words, MIC of IPM for R3 was decreased by $8 \mu \mathrm{g} / \mathrm{ml}$ EGCG from $>4$ to $0.063 \mu \mathrm{g} / \mathrm{ml}$, whereas $8 \mu \mathrm{g} / \mathrm{ml}$ of C16 inhibited the growth of R3 by itself. C16 and EGCG exhibited negative activity with IPM or AMP against MSSA (data not shown) which was similar to that shown in Figure 5A.

\section{EFFECT OF PEPTIDOGLYCAN ON ANTISTAPHYLOCOCCAL ACTIVITY OF C16}

The bactericidal activity of EGCG against $S$. aureus was blocked by the purified peptidoglycan (Yoda et al., 2004). Effects of peptidoglycan, D-Ala-D-Ala, and LPS, on the activity of C16 against MSSA ATCC25923 and MRSA ATCC43300 were determined by changes in MIC caused by the addition of each component to the medium (Table 6). MICs of C16 for both bacterial strains were reproducible and doubled in the presence of peptidoglycan as well as MICs of glycopeptide, VAN, and TEC. However MICs of C16 did not change with the addition of D-Ala-D-Ala or LPS. In contrast, MICs of VAN and TEC increased 8- and 32-fold respectively, with the addition of D-Ala-D-Ala, the target molecule of glycopeptides in cell walls.

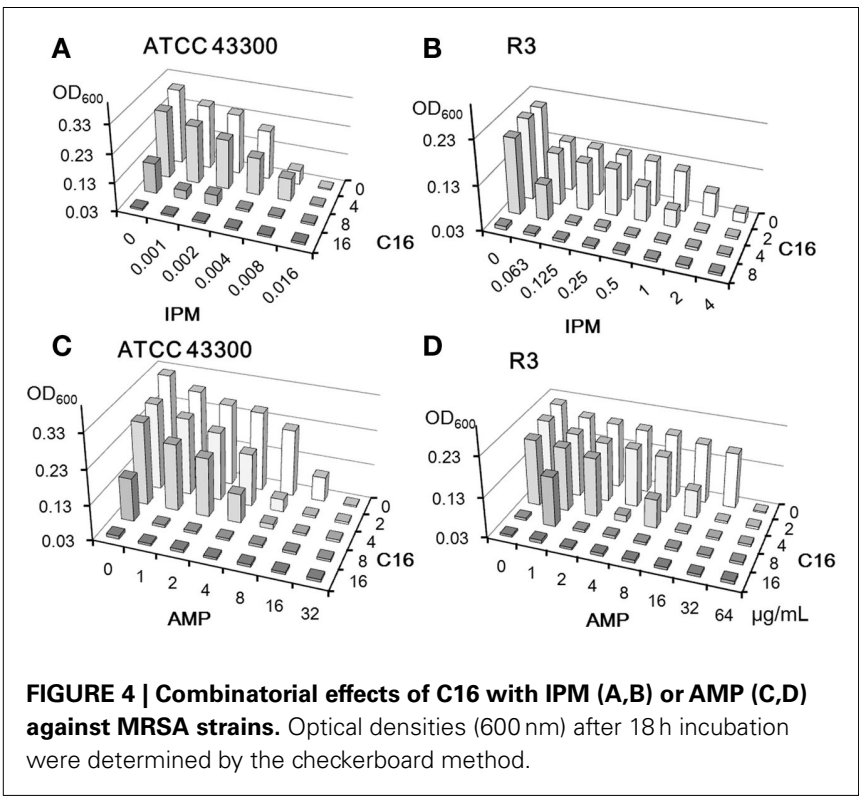

EFFECT OF EFFLUX PUMP DELETION ON ACTIVITIES OF EGCG O-ACYL DERIVATIVES IN E. COLI

Efflux pumps are major resistant factors in Gram-negative bacteria (Poole et al., 1993; Nikaido, 1998; Li and Nikaido, 2004). E. coli has several pumps that effect drug resistance (Nishino and Yamaguchi, 2001). Effects of deletion of genes associated with three of these pumps, AcrAB-TolC, AcrCD-TolC, and AcrEF-TolC were evaluated. AcrAB-TolC is the most powerful pump that is constitutively expressed in E. coli and has a broad substrate profile (Nishino and Yamaguchi, 2001). To evaluate the possibility of EGCG and its derivatives to act as a substrate for efflux pumps, MICs of the EGCG derivatives for efflux pump gene deletion mutants of $E$. coli MG1655 were determined (Table 7). MICs of all tested derivatives and EGCG for $\triangle a c r B$ and $\triangle$ tolC were lower than those for the wild-type strain. The $a c r D$ or $a c r E F$ deletion had almost no effect on the activities of the EGCG derivatives against E. coli. 

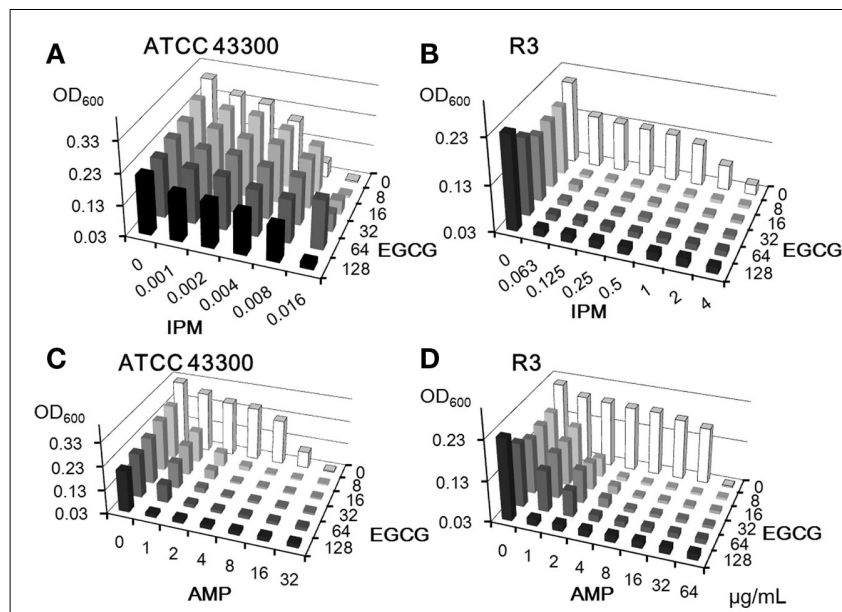

FIGURE 5 | Combinatorial effects of EGCG with IPM (A,B) or AMP (C,D) against MRSA strains. Optical densities $(600 \mathrm{~nm})$ after $18 \mathrm{~h}$ incubation were determined by the checkerboard method.

Table 6 | Effect of peptidoglycan, D-Ala-D-Ala, and lipopolysaccharide on antistaphylococcal activity of C16.

\begin{tabular}{|c|c|c|c|c|c|c|c|}
\hline \multirow[t]{2}{*}{ Agents } & \multicolumn{4}{|c|}{ ATCC25923 (MIC: $\mu \mathrm{g} / \mathrm{ml}$ ) } & \multicolumn{3}{|c|}{ ATCC43300 (MIC: $\mu \mathrm{g} / \mathrm{ml}$} \\
\hline & control & PG & D-Ala & LPS & control & PG & D-Ala \\
\hline C16 & 16 & 32 & 16 & 16 & 8 & 16 & 8 \\
\hline VAN & 2 & 4 & 16 & 2 & 1 & 2 & 16 \\
\hline TEC & 1 & 2 & 32 & 1 & 0.5 & 1 & 16 \\
\hline AMP & 0.25 & 0.25 & 0.25 & 0.25 & 16 & 8 & 16 \\
\hline
\end{tabular}

Staphylococcus aureus ATCC25923 and methicillin-resistant S. aureus ATCC43300 were used. PG, peptidoglycan; D-Ala, D-Ala-D-Ala; LPS, lipopolysaccharide. Bold letters indicate increased MICs versus control.

EGCG and its acyl derivatives were considered to be substrates for AcrAB-TolC. Susceptibility of $\Delta t o l C$, a mutant in which all TolCdependent pumps are inactive, to the $\mathrm{C} 8 \times 2$ was fourfold higher than that to EGCG.

MEMBRANE DAMAGE DETERMINED BY SYTOX GREEN UPTAKE ASSAY The bacterial membrane is considered to be one of the most effective targets of catechins (Kajiya et al., 2004). The membrane-disrupting activities of C16 were compared with those of EGCG in MSSA ATCC25923 and MRSA ATCC43300 (Figure 6). Under this condition, a polycyclic antibacterial peptide nisin at $1 \mu \mathrm{g} / \mathrm{ml}$ significantly increased SYTOX Green uptake in both bacterial strains. In a concentration-dependent manner, C16 increased SYTOX Green uptake at $\geq 8 \mu \mathrm{g} / \mathrm{ml}$ (a half of MIC) after 15 min incubation with $S$. aureus cells, while EGCG did not induce SYTOX Green uptake at $128 \mu \mathrm{g} / \mathrm{ml}$. EGCG at $64 \mu \mathrm{g} / \mathrm{ml}$ actually exhibited a protective effect; SYTOX Green uptake was less than a half of the dye uptake observed with controls. C16 disrupted the membrane of $S$. aureus more effectively than EGCG, which supports the previously observed low MIC of C16 for $S$. aureus.
Table 7 | Activities of EGCG derivatives against efflux pump gene deletion mutants of $E$. coli.

\begin{tabular}{lccccc}
\hline \multirow{2}{*}{ Strains } & \multicolumn{5}{c}{ EGCG and its derivatives (MIC: $\boldsymbol{\mu g} \mathbf{g m l})$} \\
\cline { 2 - 6 } & $\mathbf{C 8 \times \mathbf { 2 }}$ & $\mathbf{C 1 6}$ & $\mathbf{C 1 6 E}$ & $\mathbf{C 1 8 T E}$ & EGCG \\
\hline MG1655 (Wild-type) & $>128$ & $>128$ & $>128$ & $>128$ & $>256$ \\
$\Delta$ acrB & 64 & 64 & 64 & 64 & 128 \\
$\Delta$ tolC & 32 & 64 & 64 & 64 & 128 \\
$\Delta$ acrD & $>128$ & $>128$ & $>128$ & $>128$ & $>256$ \\
AacrEF & $>128$ & $>128$ & $>128$ & $>128$ & $>256$ \\
\hline
\end{tabular}

Escherichia coli MG1655 and its efflux pump gene deletion mutants were used

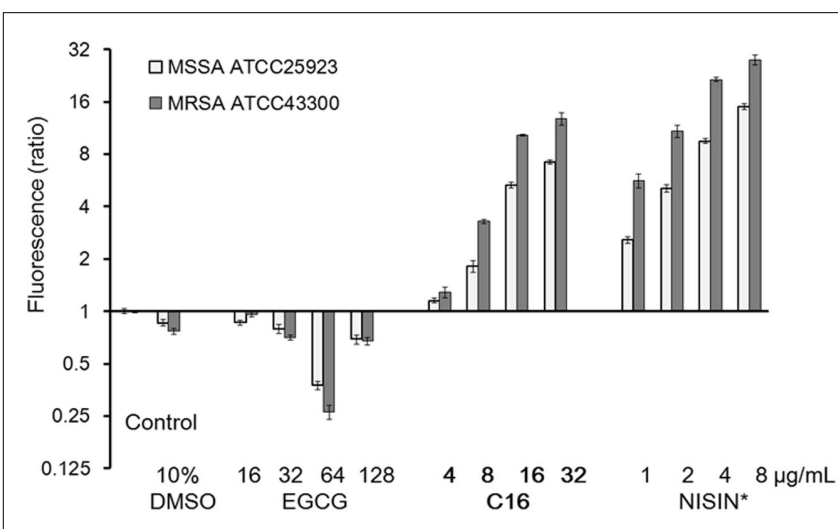

FIGURE 6 | Membrane damage caused by C16 determined by SYTOX Green uptake assay. Fluorescence was determined (Ex/Em: 504/523 nm) after $10 \mathrm{~min}$ incubation with SYTOX Green in a black 384-well plate using a microplate reader after incubation with the antimicrobial compound for $15 \mathrm{~min}$. Fluorescence is shown as a ratio to that of control. Data represent Mean $\mp$ SD $(n=3) .{ }^{*} A$ polycyclic antibacterial peptide.

\section{DISCUSSION}

Staphylococcus aureus is one of the most sensitive bacteria to tea polyphenols. The rapid bactericidal activity of tea extracts against S. aureus was demonstrated in 1989 (Toda et al., 1989), and the mechanism underlying the killing activity of catechin by disrupting the lipid bilayer of the membrane (Ikigai et al., 1993). Subsequently, interaction of catechin with lipid bilayers has been studied in detail (Kumazawa et al., 2004; Uekusa et al., 2007; Kajiya et al., 2008; Kamihira et al., 2008; Sirk et al., 2008, 2009, 2011; Sun et al., 2009). Previously, Kajiya et al. synthesized (+)-Catechin derivatives with different alkyl chain lengths and found the antibacterial activity increased markedly with elongation of the alkyl chain lengths of the derivatives and reached a maximum at a chain of four to seven carbons. Lipophilicity and the membrane-disrupting ability of these derivatives correlate with their antibacterial activities against Gram-positive organisms (Kajiya et al., 2004). In our study, addition of acyl chains longer than C12 to EGCG significantly enhanced its antibacterial activities particularly against $S$. aureus including MRSA. However, the activity of EGCG $O$-acyl derivatives decreased in the derivatives with an acyl chain length longer than $\mathrm{C} 18$, so that $\mathrm{C} 12$ and $\mathrm{C} 16$ had the highest activity against S. aureus (Figure 2). MICs of $\mathrm{C} 8 \times 2$ for the 17 S. aureus 
strains were the lowest and comparable to those of C16 (Table 2). The increased activities of these derivatives against $S$. aureus are probably a result of their enhanced affinity of their long acyl chains to interact with membranes. In fact, $8 \mu \mathrm{g} / \mathrm{ml}$ of $\mathrm{C} 16$ showed significant membrane-permeabilizing activity in $S$. aureus. On the other hand, EGCG inhibited SYTOX Green uptake to less than a half of the control at $64 \mu \mathrm{g} / \mathrm{ml}$ (Figure 6). As an explanation of the opposite effect of $64 \mu \mathrm{g} / \mathrm{ml}$ EGCG in SYTOX Green uptake assay, peptidoglycan-binding activity of EGCG could be higher than its affinity to membrane and interfered penetration of SYTOX Green into Staphylococcal cells. SYTOX Green would be permeabilized by EGCG in concentrations higher than its MIC. Against influenza virus, $\mathrm{C} 16$ also exhibited the strongest activity, suggesting an interaction with the viral membrane (Mori et al., 2008); however, the derivatives with acyl chains longer than 16 exhibited decreased activities probably because of their decreased solubility. The role of decreased solubility as being a possible reason was deduced from the higher lipophilicity speculated from the higher $\log P$ values in C18 and C20 (Table 1). Introduction of an unsaturated bond into the acyl chain tended to reduce lipophilicity and recover the activity, although it was not very effective in C16 (Table 2). Anyway, influenza virus is more sensitive to $\mathrm{C} 16$ than S. aureus and E. coli. The effect of acyl chain length on antistaphylococcal activity of EGCG was similar but not coincident with that on anti-influenza virus activity of it. The difference might come from the different surface structure of these pathogens such as cell wall in S. aureus rather than common lipid bilayer. Outer membrane and cell wall of bacteria might protect lipid bilayer from polyphenols. Acylation might possible to enhance interaction of EGCG with lipid bilayer but not break through outer membrane.

Synergistic activities of EGCG with $\beta$-lactams against MRSA have been studied previously (Hu et al., 2001, 2002a). Contemporary, EGCG is shown to have no effect on penicillin-binding protein $2^{\prime}$ (PBP2'; protein causative of methicillin-resistance in MRSA) mRNA expression or PBP2' production (Zhao et al., 2001). In combinatorial effects with other cell wall synthesis inhibitors, EGCG also enhance activity of DL-cycloserine (Zhao et al., 2001), but antagonizes the activity of VAN and TEC against MRSA (Hu et al., 2002b). Furthermore, EGCG antagonizes the activity of polymyxin B against E. coli (Hu et al., 2002b). EGCG interferes with cell wall integrity by directly binding to peptidoglycan, and this might be the major reason for the synergism between EGCG and $\beta$-lactams. EGCG might also bind directly to glycopeptides and then interfere with their activities ( $\mathrm{Hu}$ et al., 2002b). In our results, C16 activity against S. aureus was decreased with the addition of peptidoglycan, but not of D-Ala-DAla (Table 6), which indicates that $\mathrm{C} 16$ binding to peptidoglycan occurs because of some peptidoglycan structural moieties other than D-Ala-D-Ala. The peptidoglycan-binding ability of C16 without a D-Ala-D-Ala-mediated mechanism could adversely affect its antibacterial activity against $S$. aureus. In fact, $64 \mu \mathrm{g} / \mathrm{ml}$ of EGCG decreased SYTOX Green uptake in S. aureus (Figure 5), which indicates a potential protective effect of EGCG on the surface of bacteria. Binding of EGCG to peptidoglycan might interfere with binding of glycopeptides to $\mathrm{D}$-Ala-D-Ala residue. In addition, EGCG inhibits the activity of various $\beta$-lactamases not only from $S$. aureus but also from Gram-negative bacteria, including carbapenem-hydrolyzing enzyme, IMP-1 (Zhao et al., 2002, 2003). However, most cephalosporins and carbapenems are stable against penicillinase from $S$. aureus. For this reason, $\beta$-lactamase-inactivating activity of EGCG cannot explain the mechanism of synergistic activity between EGCG and IPM against MRSA. When we focus on the action mechanism of $\beta$-lactam, there are four different cell wall synthesizing enzymes in S. aureus. PBP2' alone might not completely fulfill the functions of these other intrinsic S. aureus PBPs (Zapun et al., 2008). Actually, synergism between carbapenems and other $\beta$-lactams having different PBP affinities against MRSA has also been reported (Sumita and Mitsuhashi, 1991). Cell wall synthesis partially affected by $\beta$-lactam would provide an easy access for EGCG to the membrane of MRSA. In contrast, C16 appears to access the membrane more easily than EGCG (Figure 6). Therefore, the combinatorial effect of $\beta$-lactam with C16 against MRSA might be weak. In fact, the combinatorial effects of C16 with AMP and IPM (Figure 3) were mostly within additive category (FIC indices $>0.5$ ), and were not as strong as those of EGCG with AMP (Figure 5).

Different EGCG susceptibility of Staphylococci and Gramnegative rods are observed due to the different EGCG affinities toward various cell wall components (Yoda et al., 2004). EGCG activity against Enterobacteriaceae was weak and was not strongly enhanced by acylation (Table 4); however, we did detect higher activity of the EGCG fatty acid derivatives compared to EGCG against $a c r B$ or tolC deletion mutants of MG1655 (Table 7). AcrAB-TolC can export various compounds with very different structures (Nishino and Yamaguchi, 2001). EGCG and its derivatives are now identified as substrates for AcrAB-TolC. Efflux and low outer membrane permeability could be a strong reason for the weaker activity of EGCG against Gram-negative bacteria such as E. coli. In higher concentrations, tea polyphenols can inhibit the growth of $P$. aeruginosa by permeabilizing its membrane (Yi et al., 2010). In this study, $64 \mu \mathrm{g} / \mathrm{ml}$ of C16 inhibited the growth of $P$. aeruginosa PAO1, although it was not bactericidal (Figure 3).

Acylation of EGCG enhanced its antimicrobial activity against various species of bacteria and fungi although it did not expand antibacterial spectrum (Tables 2-5). C8 $\times 2$ and C16 had the strongest activity against bacteria, particularly against $S$. aureus including MRSA. Moreover, C16 is proved to have superior activity including antivirus (Mori et al., 2008; Kaihatsu et al., 2009) and antitumor (Matsumura et al., 2008) activities. Although effect of acylation on antitoxic property of EGCG is not yet evaluated, beneficial effect would not be expected on that because toxins are usually not associated with membrane. These findings are exciting and further research may support the finding that the broad activity of C16 could be invaluable for clinicians as an alternative disinfectant as well as an alternative treatment option to antibiotics, perhaps reducing the risk of antibiotic resistance.

\section{ACKNOWLEDGMENTS}

We would like to thank Taiyo Kagaku Co. Ltd., Tokyo, Japan for their generous donation of EGCG. This study was supported by the Grant for Promotion of Fundamental Studies in Health Sciences of the National Institute of Biomedical Innovation, Grants from the Japan Society for the Promotion of Science, and the Ministry of Education, Culture, Sports, Science and Technology of Japan. 


\section{REFERENCES}

Clinical and Laboratory Standards Institute. (2009). Methods for Dilution Antimicrobial Susceptibility Tests for Bacteria That Grow Aerobically; Approved Standard-Eighth Edition. Wayne: Clinical and Laboratory Standards Institute.

Das, D. N. (1962). Studies on the antibiotic activity of tea. J. Indian Chem. Soc. 33, 849-854.

Davis, R. W., Botstein, D., and Roth, J. R. (1980). Advanced Bacterial Genetics. Cold Spring Harbor, NY: Cold Spring Harbor Laboratory.

Friedman, M. (2007). Overview of antibacterial, antitoxin, antiviral, and antifungal activities of tea flavonoids and teas. Mol. Nutr. Food Res. 51, 116-134.

Green, R. H. (1949). Inhibition of multiplication of influenza virus by extracts of tea. Proc. Soc. Exp. Biol. Med. 71, 84.

Hara, Y., Watanabe, M., Ishigami, T., Shimamura, T., and Sakaguchi, G. (1990). Use of Tea Polyphenol(s) as Antitoxin(s) to Counteract Bacterial Exotoxin(s). Tokyo: Mitsui Norin Co. Ltd.

Hisano, M., Yamaguchi, K., Inoue, Y., Ikeda, Y., Iijima, M., Adachi, M., and Shimamura, T. (2003). Inhibitory effect of catechin against the superantigen staphylococcal enterotoxin B (SEB). Arch. Dermatol. Res. 295, 183-189.

Hu, Z. Q., Zhao, W. H., Asano, N., Yoda, Y., Hara, Y., and Shimamura, T. (2002a). Epigallocatechin gallate synergistically enhances the activity of carbapenems against methicillinresistant Staphylococcus aureus. Antimicrob. Agents Chemother. 46, 558-560.

Hu, Z. Q., Zhao, W. H., Yoda, Y., Asano, N., Hara, Y., and Shimamura, T. (2002b). Additive, indifferent and antagonistic effects in combinations of epigallocatechin gallate with 12 non-beta-lactam antibiotics against methicillin-resistant Staphylococcus aureus. J. Antimicrob. Chemother. 50, 1051-1054.

Hu, Z. Q., Zhao, W. H., Hara, Y., and Shimamura, T. (2001). Epigallocatechin gallate synergy with ampicillin/sulbactam against 28 clinical isolates of methicillin-resistant Staphylococcus aureus. J. Antimicrob. Chemother. 48, 361-364.

Ikigai, H., Nakae, T., Hara, Y., and Shimamura, T. (1993). Bactericidal catechins damage the lipid bilayer. Biochim. Biophys. Acta 1147, 132-136.

Kaihatsu, K., Mori, S., Matsumura, H., Daidoji, T., Kawakami, C., Kurata,
H., Nakaya, T., and Kato, N. (2009). Broad and potent anti-influenza virus spectrum of epigallocatechin3-O-gallate-monopalmitate. J. Mol. Genet. Med. 3, 195-197.

Kajiya, K., Hojo, H., Suzuki, M., Nanjo, F., Kumazawa, S., and Nakayama, T. (2004). Relationship between antibacterial activity of $(+)$-catechin derivatives and their interaction with a model membrane. J. Agric. Food Chem. 52, 1514-1519.

Kajiya, K., Kumazawa, S., Naito, A., and Nakayama, T. (2008). Solid-state NMR analysis of the orientation and dynamics of epigallocatechin gallate, a green tea polyphenol, incorporated into lipid bilayers. Magn. Reson. Chem. 46, 174-177.

Kamihira, M., Nakazawa, H., Kira, A., Mizutani, Y., Nakamura, M., and Nakayama, T. (2008). Interaction of tea catechins with lipid bilayers investigated by a quartzcrystal microbalance analysis. Biosci. Biotechnol. Biochem. 72, 1372-1375.

Kumazawa, S., Kajiya, K., Naito, A., Saito, H., Tuzi, S., Tanio, M., Suzuki, M., Nanjo, F., Suzuki, E., and Nakayama, T. (2004). Direct evidence of interaction of a green tea polyphenol, epigallocatechin gallate, with lipid bilayers by solidstate nuclear magnetic resonance. Biosci. Biotechnol. Biochem. 68, 1743-1747.

Li, X. Z., and Nikaido, H. (2004). Effluxmediated drug resistance in bacteria. Drugs 64, 159-204.

Martinez-Irujo, J. J., Villahermosa, M. L., Alberdi, E., and Santiago, E. (1996). A checkerboard method to evaluate interactions between drugs. Biochem. Pharmacol. 51, 635-644.

Matsumoto, Y., Hayama, K., Sakakihara, S., Nishino, K., Noji, H., Iino, R., and Yamaguchi, A. (2011). Evaluation of multidrug efflux pump inhibitors by a new method using microfluidic channels. PLOS ONE 6, e18547. doi: 10.1371/journal.pone. 0018547

Matsumura, K., Kaihatsu, K., Mori, S., Cho, H. H., Kato, N., and Hyon, S. H. (2008). Enhanced antitumor activities of (-)-epigallocatechin3-O-gallate fatty acid monoester derivatives in vitro and in vivo. Biochem. Biophys. Res. Commun. 377, 1118-1122.

Mori, S., Miyake, S., Kobe, T., Nakaya, T., Fuller, S. D., Kato, N., and Kaihatsu, K. (2008). Enhanced anti-influenza A virus activity of (-)-epigallocatechin-3-O-gallate fatty acid monoester derivatives: effect of alkyl chain length. Bioorg. Med. Chem. Lett. 18, 4249-4252.

Mukhtar, H., Wang, Z. Y., Katiyar, S. K., and Agarwal, R. (1992). Tea components: antimutagenic and anticarcinogenic effects. Prev. Med. 21, 351-360.

Mukoyama, A., Ushijima, H., Nishimura, S., Koike, H., Toda, M., Hara, Y., and Shimamura, T. (1991). Inhibition of rotavirus and enterovirus infections by tea extracts. Jpn. J. Med. Sci. Biol. 44 181-186.

Nakayama, M., Suzuki, K., Toda, M., Okubo, S., Hara, Y., and Shimamura, T. (1993). Inhibition of the infectivity of influenza virus by tea polyphenols. Antiviral Res. 21, 289-299.

Nikaido, H. (1998). Antibiotic resistance caused by Gram-negative multidrug efflux pumps. Clin. Infect. Dis. 27, S32-S41.

Nishino, K., Senda, Y., and Yamaguchi, A. (2008). The AraC-family regulator GadX enhances multidrug resistance in Escherichia coli by activating expression of mdtEF multidrug efflux genes. J. Infect. Chemother. 14, 23-29.

Nishino, K., and Yamaguchi, A. (2001). Analysis of a complete library of putative drug transporter genes in Escherichia coli. J. Bacteriol. 183 5803-5812.

Poole, K., Krebes, K., McNally, C., and Neshat, S. (1993). Multiple antibiotic resistance in Pseudomonas aeruginosa: evidence for involvement of an efflux operon. J. Bacteriol. 175, 7363-7372.

Shimamura, T. (2009). Catechinology. Tokyo: Microbiology, Showa University School of Medicine.

Shimamura, T., and Hara, Y. (1997). Composition for Treating CNS Tumours by Addition of a Synergistic Poly:Phenolic Chroman Derivatives which is a Catechin or Theaflavin cpd. Tokyo: Mitsui Norin Co. Ltd.

Sirk, T. W., Brown, E. F., Friedman, M., and Sum, A. K. (2009). Molecular binding of catechins to biomembranes: relationship to biological activity. J. Agric. Food Chem. 57, 6720-6728.

Sirk, T. W., Brown, E. F., Sum, A. K. and Friedman, M. (2008). Molecular dynamics study on the biophysical interactions of seven green tea catechins with lipid bilayers of cell membranes. J. Agric. Food Chem. 56, 7750-7758.

Sirk, T. W., Friedman, M., and Brown, E. F. (2011). Molecular binding of black tea theaflavins to biological membranes: relationship to bioactivities. J. Agric. Food Chem. 59, 3780-3787.

Sumita, Y., and Mitsuhashi, S. (1991). In vitro synergistic activity between meropenem and other betalactams against methicillin-resistant Staphylococcus aureus. Eur. J. Clin. Microbiol. Infect. Dis. 10, 77-84.

Sun, Y., Hung, W. C., Chen, F. Y., Lee, C. C., and Huang, H. W. (2009). Interaction of tea catechin (-)epigallocatechin gallate with lipid bilayers. Biophys. J. 96, 1026-1035.

Toda, M., Okubo, S., Hiyoshi, R., and Shimamura, T. (1989). The bactericidal activity of tea and coffee. Lett. Appl. Microbiol. 8, 123-125.

Toda, M., Okubo, S., Ikigai, H., and Shimamura, T. (1990). Antibacterial and anti-hemolysin activities of tea catechins and their structural relatives. Nihon Saikingaku Zasshi 45 , 561-566.

Uekusa, Y., Kamihira, M., and Nakayama, T. (2007). Dynamic behavior of tea catechins interacting with lipid membranes as determined by NMR spectroscopy. $J$. Agric. Food Chem. 55, 9986-9992.

Xiao, X., Yang, Z. Q., Shi, L. Q., Liu, J., and Chen, W. (2008). Antiviral effect of epigallocatechin gallate (EGCG) on influenza A virus. Zhongguo Zhong Yao Za Zhi 33, 2678-2682.

Yam, T. S., Shah, S., and HamiltonMiller, J. M. (1997). Microbiological activity of whole and fractionated crude extracts of tea (Camellia sinensis), and of tea components. FEMS Microbiol. Lett. 152, 169-174.

Yamaguchi, K., Honda, M., Ikigai, H., Hara, Y., and Shimamura, T. (2002). Inhibitory effects of (-)epigallocatechin gallate on the life cycle of human immunodeficiency virus type 1 (HIV-1). Antiviral Res. 53, 19-34.

Yi, S. M., Zhu, J. L., Fu, L. L., and Li, J. R. (2010). Tea polyphenols inhibit Pseudomonas aeruginosa through damage to the cell membrane. Int. J. Food Microbiol. 144, 111-117.

Yoda, Y., Hu, Z. Q., Zhao, W. H., and Shimamura, T. (2004). Different susceptibilities of Staphylococcus and Gram-negative rods to epigallocatechin gallate. J. Infect. Chemother. 10, 55-58.

Zapun, A., Contreras-Martel, C., and Vernet, T. (2008). Penicillin-binding proteins and beta-lactam resistance. FEMS Microbiol. Rev. 32, 361-385.

Zhao, W. H., Asano, N., Hu, Z. Q., and Shimamura, T. (2003). Restoration of antibacterial activity of beta-lactams by epigallocatechin 
gallate against beta-lactamaseproducing species depending on location of beta-lactamase. J. Pharm. Pharmacol. 55, 735-740.

Zhao, W. H., Hu, Z. Q., Hara, Y., and Shimamura, T. (2002). Inhibition of penicillinase by epigallocatechin gallate resulting in restoration of antibacterial activity of penicillin against penicillinaseproducing Staphylococcus aureus. Antimicrob. Agents Chemother. 46, 2266-2268.
Zhao, W. H., Hu, Z. Q., Okubo, S., Hara, Y., and Shimamura, T. (2001). Mechanism of synergy between epigallocatechin gallate and beta-lactams against methicillinresistant Staphylococcus aureus. Antimicrob. Agents Chemother. 45, 1737-1742.

Conflict of Interest Statement: The authors declare that the research was conducted in the absence of any commercial or financial relationships that could be construed as a potential conflict of interest.

Received: 17 October 2011; accepted: 31 January 2012; published online: 16 February 2012.

Citation: Matsumoto $Y$, Kaihatsu K, Nishino K, Ogawa M, Kato $N$ and Yamaguchi A (2012) Antibacterial and antifungal activities of new acylated derivatives of epigallocatechin gallate. Front. Microbio. 3:53. doi 10.3389/fmicb.2012.00053
This article was submitted to Frontiers in Antimicrobials, Resistance and Chemotherapy, a specialty of Frontiers in Microbiology.

Copyright () 2012 Matsumoto, Kaihatsu, Nishino, Ogawa, Kato and Yamaguchi. This is an open-access article distributed under the terms of the Creative Commons Attribution Non Commercial License, which permits non-commercial use, distribution, and reproduction in other forums, provided the original authors and source are credited. 